\title{
Decisiones de financiamiento en empresas del sector de alojamiento y servicios de comida: Trade Off vs. Pecking Order
}

\section{Financing decisions in food service and accommodation companies: Trade Off vs. Pecking Order}

\author{
Ana Luisa Carvajal-Salgado ${ }^{1 *}$, Christel Alexandra Michilena Chila ${ }^{1}$ y Gustavo Adolfo Acuña Corredor ${ }^{2}$ \\ ${ }^{1}$ Universidad Espíritu Santo \\ ${ }^{2}$ Universidad Nacional de Colombia \\ *anacs18luisa@hotmail.com
}

DOI: https://doi.org/10.26871/killkana_social.v2i4.96

\begin{abstract}
Resumen
Este artículo tiene como propósito determinar la fuente de financiación utilizada por las empresas del sector de alojamiento y servicios de comida, tomando como marco de enfoque las teorías Pecking Order y Trade Off, en el periodo 20132015, años a través de los cuales se estudia la situación actual de este sector. Para la investigación se utilizó información proveniente del estado de situación financiera y del estado de resultados de las empresas ecuatorianas del sector de alojamiento y servicios de comida, información comparable porque está elaborada de acuerdo con las normas contables. Se realizó una investigación cuantitativa de tipo explicativo, longitudinal; la población estuvo conformada por 614 empresas activas que reportan sus estados financieros a la Superintendencia de Compañías del Ecuador. Se utiliza como metodología el análisis financiero y económico a través de los indicadores de endeudamiento, apalancamiento y rentabilidad. Los resultados muestran que las empresas se financian con recursos propios, con alta concentración en el corto plazo y en menor medida con instituciones financieras, y se evidencia que el tamaño de la empresa es un factor que influye al momento de obtener recursos, corroborando la hipótesis que establece que las empresas se financian de acuerdo con la teoría del Pecking Order, sin embargo, las grandes empresas acuden en mayor proporción a deuda bancaria al contrario de la tendencia de las pequeñas empresas, ya que a las instituciones financiera muchas veces prefieren otorgar recursos monetarios a aquellas empresas que tengan mayor garantía para cubrir sus préstamos, lo cual ocasiona desigualdad empresarial.
\end{abstract}

Palabras clave: Decisiones de financiamiento, trade off, pecking order, tamaño de las empresas, sector alojamiento y servicios de comida.

\begin{abstract}
The purpose of this article is to determine the source of funding used by companies in the sector of food accommodation and services, using the Pecking Order and Trade Off theories in the period 2013-2015. For the investigation, information was used from the statement of financial position and income statement of the Ecuadorian companies in the accommodation and food services sector, comparable information because it is prepared in accordance with accounting standards. A quantitative investigation of explanatory, longitudinal type was carried out; the population was formed by 614 active companies that report their financial statements to the Superintendencia de Compañias del Ecuador. Financial and economic analysis is used as a methodology through the indicators of indebtedness, leverage and profitability. The results show that companies are financed with their own resources, with high concentration in the short term and to a lesser extent with financial institutions, and it is evident that the size of the company is a factor that influences when obtaining resources, corroborating the hypothesis that establishes that companies are financed according to the theory of the Pecking Order, however, large companies go in greater proportion to bank debt contrary to the trend of small businesses, since financial institutions often prefer to grant resources monetary benefits to those companies that have the greatest guarantee to cover their loans, which causes business inequality.
\end{abstract}

Key words: Financing decisions, trade off, pecking order, size of companies, sector accommodation and food services. 


\section{Introducción}

El análisis de las decisiones financieras es objeto de amplia controversia, debido a la importancia de determinar los niveles de estructura de capital-recursos propios y ajenos-que generen valor a la firma.

Los primeros estudios fueron planteados en 1958 con la tesis de irrelevancia propuesta por Modigliani y Miller, donde mencionan que "en una economía de mercado perfecta, en la que no hay costos de transacción e impuestos, la manera en que se financian las inversiones de las empresas no afecta su valor" (p. 261). Sin embargo, está claro que los mercados financieros no pueden delimitarse de esta manera. Como argumentan Barona y Gómez (2010), Portal, Zani y Da Silva (2012), Zata-Poutziouris, P. (2011), los costos de transacción son ciertamente limitaciones reales que las empresas deben enfrentar al elegir su estrategia de financiamiento en las diferentes etapas de crecimiento, esta decisión a la vez debe de contribuir a minimizar el costo de capital y maximizar el valor de la empresa.

En la actualidad, desde el punto de vista de los mercados imperfectos, este tema es objeto de análisis en el ámbito de las finanzas, siendo el modelo de Pecking Order, también llamado de Jerarquización Financiera, y el modelo Trade Off, conocido como apalancamiento objetivo, las más estudiadas.

La teoría de Pecking Order cuyo principal referente es Myers y Majluf, (1984) señala que las empresas utilizarán para financiar sus proyectos, en primer lugar sus resultados económicos (autofinanciación), posteriormente la emisión de deuda y como último recurso la emisión de acciones (Zambrano Vargas y Acuña Corredor, 2011), entre tanto, la teoría del Trade Off propuesto por Modigliani y Miller (1963), sostiene que las empresas buscan el apalancamiento financiero por la minimización de las obligaciones fiscales, debido a que los intereses sobre la deuda son deducibles de impuestos.

Explorar las decisiones de financiación de las empresas del sector de alojamiento y servicios de comida, responde a distintos motivos; por un lado, Camino y Guale (2017) lo consideran: "el sector que más aporta al desarrollo del país, tanto en empleo como en crecimiento económico debido a su encadenamiento con otros sectores productivos como lo son el transporte y las actividades de comercio" (p. 83), por el otro, las diversas oportunidades de crecimiento, por el impulso y la promoción que se está dando en la actualidad al turismo ecuatoriano. Con estos datos se aprecia que las empresas objeto de estudio, para adaptarse a la evolución económica del entorno, debe aplicar estrategias de inversión que les permitan satisfacer en mayor medida las necesidades de los clientes.

Por lo anterior, es importante conocer la forma de financiación utilizada por los empresarios, debido a que estas decisiones son de gran relevancia porque además de permitir colocar recursos para su desarrollo, contribuye en la generación de valor de los diferentes establecimientos que conforman dicho sector. Aquellos antecedentes, han permitido el planteamiento del objetivo de este trabajo que es determinar la fuente de financiación utilizada por las empresas del sector de alojamiento y servicios de comida, a través de los indicadores financieros de endeudamiento y apalancamiento, tomando como marco de enfoque las teorías Pecking Order y Trade Off.

El estudio se aplicó a 614 empresas con un total de 1842 observaciones que corresponden a toda la población del sector objeto de análisis, que disponen de información sobre sus indicadores financieros, en el Portal de Información de la Superintendencia de Compañías el Ecuador, disponible a través de su página web, donde se encuentran las empresas activas del sector; el período de análisis es 2013-2015, años a través de los cuales se estudia la situación actual de este sector y se dispone de la información financiera del mayor número de empresas para los tres años de estudio.

El trabajo se divide en cuatro partes: primero una revisión literaria, en el cual constarán las teorías y conceptualizaciones más relevantes sobre el tema y estudios previos realizados sobre la estructura de capital, en la segunda sección, se describe la metodología a aplicar para el desarrollo del trabajo, en tercer lugar se encuentran los procedimientos que se aplicaron para la obtención de la información, en cuarto lugar, el análisis de los resultados y, finalmente, la conclusión del trabajo.

\section{Marco teórico o antecedentes}

\subsection{Teoría Pecking Order}

La teoría de Pecking Order, supone que no existe una estructura óptima de capital, sino que aplican una escala de jerarquías a la hora de financiarse (Zambrano Vargas y Acuña Corredor, 2011). Esta teoría afirma que las empresas seguirán el siguiente orden para financiarse: 1. Resultados económicos obtenidos por la empresa, que es la fuente más recurrida porque tiene costos más bajos que una financiación externa, 2. emisión de deuda y, como último recurso 3. emisión de acciones.

De acuerdo con esta teoría, la estructura financiera de una empresa no está dada por el nivel óptimo de deuda sino por la necesidad de financiar nuevas inversiones, considerando la estructura óptima de endeudamiento como una posibilidad cuando se han agotado los recursos internos. Kayhan y Titman citado en Rodrigues y Frois (2014), Medina, A. Salinas, J., Ochoa, L. y Molina, C. (2012), argumentan que se debe considerar que las pequeñas y medianas empresas tienen menos acceso al sistema financiero que aquellas empresas de mayor tamaño, por ello buscan financiarse con recursos propios o a través de nuevas aportaciones de los accionistas.

La política financiera de las empresas desde la postura de Pecking Order se ve afectada por la aversión al riesgo de gestión. En el caso de las pequeñas y medianas empresas los propietarios no quieren perder el control, por ello, la teoría Pecking Order se vuelve importante teniendo en cuenta los costos de la deuda (Pettit y Singer, 1985), que 
es la principal consecuencia de información asimétrica en el mercado de crédito (Barona Zuluaga y Rivera Godoy, 2012), (Mejía Amaya, 2015), (Saona Hoffmann, 2009), (Watson y Wilson, 2002). Cuanto mayor sea la información asimétrica, mayor será la restricción para obtener créditos bancarios, por tanto, se espera encontrar una menor proporción de deuda bancaria.

Medina et al. (2012), Rajan y Zingales (1995), ShyamSunder y Myers (1999), sostienen que teóricamente desde la perspectiva de Pecking Order existen factores considerados determinantes en las decisiones de financiación; entre ellos:

- Rentabilidad del activo: Este indicador está asociado a la capacidad de la empresa para generar fondos internos. A mayor rentabilidad mayor generación de fondos internos y por tanto menor necesidad de deuda. Existe una relación negativa entre endeudamiento y rentabilidad de los activos de la empresa (ShyamSunder y C. Myers, 1999)

- Tamaño: Esta variable se relaciona con la asimetría de la información, en la cual a menor tamaño mayor asimetría de la información y por tanto mayor es la preferencia de autofinanciación y mayores las dificultades para obtener fondos de largo plazo. En las pequeñas y medianas empresas la asimetría de información es mayor, las empresas se dedican a buscar fuentes de financiación que soliciten menos requisitos (Moreira Da Silva y Rodríguez Sanz, 2006), (Rajan y Zingales, 1995)

\subsection{Teoría Trade Off}

La teoría Trade off, se la conoce como la teoría de equilibrio de la estructura de capital debido a que con- sidera la existencia de una estructura óptima de capital, es decir, una combinación entre recursos propios y deuda que permite aumentar el valor de la empresa. Sin embargo, dentro de este enfoque se establece un límite al monto de endeudarse porque existen costos que anulan los beneficios fiscales de la deuda, estos son: los costos de agencia y los costos de dificultades financieras, entendiéndose a estos últimos como los costos de oportunidad, gastos contables, administrativos o legales que se generan en caso de quiebra de la empresa (Zambrano Vargas y Acuña Corredor, 2011).

Se debe considerar que si los niveles de deuda adquiridos por la empresa son demasiado elevados existe la posibilidad que la empresa no se encuentre en posición de cumplir con sus compromisos, esta situación generaría a más de lo mencionado mayores probabilidades de quiebra por la mala gestión financiera. Como indican Isaac, Flores y Jaramillo (2010), el análisis de los costos y beneficios de recurrir a deuda llevan a las empresas sobre-endeudadas a reducir su razón de deuda, y a las sub-endeudadas a incrementarlo.

Moreira y Rodríguez (2006), manifiesta que la teoría Trade Off se ve afectada por el crecimiento en ventas, debido a que las empresas que mantienen un crecimiento constante en sus ventas casi siempre necesitan invertir en activos fijos, existiendo una relación positiva entre endeudamiento y crecimiento. Por su parte, Jensen (1986), argumenta que las empresas adquieren deuda para evitar que los gerentes utilicen inadecuadamente los flujos de caja libre, existiendo una relación positiva entre liquidez y endeudamiento.

A nivel de evidencia empírica, en la Tabla 1, se muestran algunos estudios previos realizados por varios autores que han analizado la estructura financiera con la Teoría Pecking Order y Trade Off.

Tabla 1: Resultados de investigaciones.

\begin{tabular}{|c|c|c|c|}
\hline Autor & Año & Objeto de estudio & Resultados \\
\hline $\begin{array}{l}\text { Shyam- } \\
\text { Sunder y } \\
\text { Myers }\end{array}$ & 1999 & $\begin{array}{l}157 \text { empresas del sector } \\
\text { industrial para el periodo } \\
1971-1989\end{array}$ & $\begin{array}{l}\text { Los resultados indican que las empresas usan deuda para } \\
\text { financiar sus requerimientos de efectivo, presentando mayor } \\
\text { nivel de aplicación a la teoría Pecking Order. }\end{array}$ \\
\hline $\begin{array}{l}\text { Aybar, Ca- } \\
\text { sino y Ló- } \\
\text { pez }\end{array}$ & 2001 & $\begin{array}{l}\text { Empresas españolas } \\
\text { pequeñas, medianas } \\
\text { y grandes del sector } \\
\text { manufactura para el } \\
\text { periodo 1994-1998 }\end{array}$ & $\begin{array}{l}\text { Analizan los determinantes del endeudamiento y su relación } \\
\text { positiva con la liquidez, intensidad del capital y crecimiento } \\
\text { de las ventas, comprobando la evidencia del Pecking Order, } \\
\text { al indicar que existe menos probabilidad de quiera, mayor } \\
\text { capacidad de generar recursos internos. Comprueban que las } \\
\text { variables independientes: tamaño y edad de las empresas no } \\
\text { son confirmadas en el estudio. }\end{array}$ \\
\hline $\begin{array}{l}\text { Fama } \\
\text { French }\end{array}$ & 2002 & $\begin{array}{l}\text { Periodo } 1965-1999 \text {, em- } \\
\text { presas que presentan in- } \\
\text { formación en Compustat }\end{array}$ & $\begin{array}{l}\text { Los autores demuestran que se financian con recursos inter- } \\
\text { nos. Además, indican que existe una relación negativa entre } \\
\text { el nivel de endeudamiento y la inversión esperada y una } \\
\text { relación positiva con el tamaño de la empresa. }\end{array}$ \\
\hline
\end{tabular}


Tabla 1: Resultados de investigaciones. (continuación)

\begin{tabular}{|c|c|c|c|}
\hline Autor & Año & Objeto de estudio & Resultados \\
\hline $\begin{array}{l}\text { Lemmon } \\
\text { y Zender }\end{array}$ & 2010 & Empresas & $\begin{array}{l}\text { Comprueban fuerte evidencia del Pecking Order. Sin em- } \\
\text { bargo, sostienen que, en ausencia de preocupaciones sobre } \\
\text { la capacidad de endeudamiento, la deuda parece preferible. } \\
\text { Estas preocupaciones explican en gran medida el uso de } \\
\text { nuevas formas de financiación como la emisión de acciones } \\
\text { considerada como última opción para financiar nuevas inver- } \\
\text { siones. }\end{array}$ \\
\hline $\begin{array}{l}\text { Cardone y } \\
\text { Casasola }\end{array}$ & 2003 & $\begin{array}{l}13.266 \text { pequeñas y me- } \\
\text { dianas empresas españo- } \\
\text { las durante el período } \\
1994-1998\end{array}$ & $\begin{array}{l}\text { Los resultados obtenidos destacan que la deuda bancaria es } \\
\text { más fácil de conseguir para las pymes con más oportunida- } \\
\text { des de crecimiento. Además, cuanto más rentables son las } \\
\text { empresas, mayores son los fondos de largo plazo obtenidos. }\end{array}$ \\
\hline $\begin{array}{l}\text { Tong } \quad y \\
\text { Green }\end{array}$ & 2004 & $\begin{array}{l}\text { Top } 50 \text { de mayores em- } \\
\text { presas chinas cotizan en } \\
2002\end{array}$ & $\begin{array}{l}\text { Analizan la teoría de Pecking Order y Trade Off. En su } \\
\text { estudio muestran una relación negativa entre apalancamiento } \\
\text { y dividendos, concluyendo que la teoría más relacionada a la } \\
\text { estructura de capital de estas empresas es la teoría de Pecking } \\
\text { Order. }\end{array}$ \\
\hline $\begin{array}{l}\text { Sarmiento } \\
\text { y Salazar }\end{array}$ & 2005 & Empresas colombianas & $\begin{array}{l}\text { Muestran en su trabajo una relación inversa con rentabilidad, } \\
\text { variabilidad de los beneficios, costo de la deuda, oportunidad } \\
\text { de crecimiento, edad de la empresa, costos de insolvencia, } \\
\text { protección fiscal; y existe una relación positiva con el tamaño } \\
\text { de la empresa }\end{array}$ \\
\hline Zamudio & 2005 & $\begin{array}{l}\text { Empresas colombianas } \\
\text { en el periodo } 2004\end{array}$ & $\begin{array}{l}\text { A través de su estudio concluyen que la fuente de financia- } \\
\text { ción mediante acciones es la menos utilizada por las em- } \\
\text { presas. Considera a la variable utilidades retenidas como la } \\
\text { principal fuente de financiación al presentar una correlación } \\
\text { positiva con la deuda financiera. }\end{array}$ \\
\hline Rivera & 2008 & $\begin{array}{l}18 \text { Pymes del sector de } \\
\text { confecciones del Valle } \\
\text { de Cauca periodo 2000- } \\
2006\end{array}$ & $\begin{array}{l}\text { La estructura de capital en las pymes está determinada de } \\
\text { forma negativa con la retención de utilidades y, con relación } \\
\text { positiva la oportunidad de crecimiento. En las medianas } \\
\text { empresas existe una relación inversa entre endeudamiento a } \\
\text { largo plazo y la retención de utilidades, costo de la deuda y } \\
\text { la protección fiscal de la deuda, mientras que en las pequeñas } \\
\text { empresas existe una correlación positiva además la edad } \\
\text { también prestan una relación directa con el endeudamiento. }\end{array}$ \\
\hline $\begin{array}{l}\text { Wadnipar } \\
\text { y Cruz }\end{array}$ & 2008 & Empresas colombianas & $\begin{array}{l}\text { Encuentran que las decisiones de financiamiento se efectúan } \\
\text { en función de la teoría Pecking Order. Contrastan el finan- } \\
\text { ciamiento con la oportunidad de crecimiento, el déficit de } \\
\text { financiamiento, la tangibilidad de los activos y el tamaño de } \\
\text { las empresas, (a mayor tamaño mayor acceso al mercado de } \\
\text { capitales). }\end{array}$ \\
\hline $\begin{array}{l}\text { Frank } \\
\text { Goyal }\end{array}$ & 2009 & $\begin{array}{l}\text { Empresas estadouniden- } \\
\text { ses que cotizan en bolsa } \\
\text { desde } 1950 \text { hasta } 2003 \text {. }\end{array}$ & $\begin{array}{l}\text { Encuentran que los factores más fiables para explicar el apa- } \\
\text { lancamiento son: la influencia de la industria, la tangibilidad } \\
\text { de los activos y, el indicador de activo que tienen efec- } \\
\text { to positivo en el apalancamiento, en contraparte, el índice } \\
\text { de inflación tiene relación negativa con el apalancamiento. } \\
\text { Además, encuentran que las empresas que pagan dividendos } \\
\text { tienden a tener un menor apalancamiento. Sin embargo, el } \\
\text { impacto del tamaño de la empresa, la relación mercado-libro, } \\
\text { y el efecto de la inflación no son confiables, para medir } \\
\text { el nivel de para el apalancamiento. La evidencia empírica } \\
\text { parece razonablemente consistente con algunas versiones de } \\
\text { la teoría del Trade-off. }\end{array}$ \\
\hline
\end{tabular}


Tabla 1: Resultados de investigaciones. (continuación)

\begin{tabular}{|c|c|c|c|}
\hline Autor & Año & Objeto de estudio & Resultados \\
\hline $\begin{array}{l}\text { Medina, } \\
\text { Jaramillo } \\
\text { y Rojas }\end{array}$ & 2011 & $\begin{array}{l}117 \text { empresas textiles del } \\
\text { Valle de Aburra para } \\
2005-2009\end{array}$ & $\begin{array}{l}\text { Sostienen que la principal fuente de financiación proviene de } \\
\text { recursos internos. Estas empresas se financian con fuentes a } \\
\text { corto plazo }\end{array}$ \\
\hline $\begin{array}{l}\text { Medina, } \\
\text { Salinas, } \\
\text { Ochoa, } \\
\text { Molina }\end{array}$ & 2012 & $\begin{array}{l}\text { Empresas manufacture- } \\
\text { ras colombianas, periodo } \\
2005-2011\end{array}$ & $\begin{array}{l}\text { Estudia el Pecking Order, como opción de financiación, } \\
\text { para ello considera los indicadores de rentabilidad, déficit } \\
\text { de financiación y opciones de crecimiento como variables } \\
\text { determinantes que influyen en el nivel endeudamiento por su } \\
\text { correlación positiva. }\end{array}$ \\
\hline Carvajal & 2013 & $\begin{array}{l}\text { Sector infraestructura y } \\
\text { obra civil }\end{array}$ & $\begin{array}{l}\text { Estudia el Flujo de caja libre y costo promedio ponderado } \\
\text { de capital como factores claves para la estructura de capital. } \\
\text { Los resultados indican que la estructura de capital no es } \\
\text { estática en el tiempo, esta decisión se soporta en función } \\
\text { de los beneficios fiscales, costos de dificultades financieras } \\
\text { y sostenibilidad del activo financiero. }\end{array}$ \\
\hline $\begin{array}{l}\text { Amponsah, } \\
\text { Nyarko- } \\
\text { Baasi, } \\
\text { Hughes }\end{array}$ & 2013 & $\begin{array}{l}\text { Empresas que cotizan en } \\
\text { Ghana durante el período } \\
\text { de cinco años compren- } \\
\text { dido entre } 2005 \text { y } 2009\end{array}$ & $\begin{array}{l}\text { Obtuvo como resultado de que existen interrelaciones po- } \\
\text { sitivas entre deuda a corto plazo y ROE y muestra que las } \\
\text { empresas que ganan mucho utilizan más deuda a corto plazo } \\
\text { para financiarse. En otras palabras, la deuda a corto plazo es } \\
\text { una fuente de financiación a favor de las empresas ghanesas, } \\
\text { al representar el } 85 \% \text { del total de la deuda financiada. Los } \\
\text { resultados de la regresión demostraron que existe una rela- } \\
\text { ción positiva entre el total de la deuda y el ROE que miden } \\
\text { la relación entre deuda total y rentabilidad. Esto indica que } \\
\text { las empresas que ganan mucho dependen de la deuda como } \\
\text { su opción de financiación clave. }\end{array}$ \\
\hline
\end{tabular}

Fuente: Elaboración Propia, con base en la revisión y análisis de la bibliografía

\section{Metodología}

Para el desarrollo de la metodología se tomó como referencia el trabajo de Rivera, (2007)

Se realizó una investigación cuantitativa, de tipo explicativo, que intenta determinar las tendencias de financiamiento de las empresas del sector alojamiento y servicios de comida del Ecuador. La investigación explicativa involucra un estudio descriptivo, que permitirá analizar las variables a desarrollarse.

En el trabajo se aplicó un diseño de investigación documental, debido a que la información que se analizó fue recopilada de la base de datos de indicadores financieros del Portal de Información de la Superintendencia de Compañías del Ecuador, a través de su página web. (Consultado en junio 26, 2017 de http://appscvs.supercias.gob.ec)

El estudio realizado es no experimental, longitudinal, en el se utiliza un panel de las empresas que disponen de información de los indicadores financieros, en los períodos 2013-2015.

Se identificó una población de 614 empresas a nivel pais, con un total de 1842 observaciones, luego de depurar la base de datos de la Supercía, para lo cual se identificarón los siguientes criterios; empresas:

1) Consideradas como activas por la Superintendencia de Compañías del Ecuador, como ente regular.
2) Que reportan información de sus cuentas contables a través de los estados financieros para los tres años de estudio, 2013-2015.

3) Que para los tres años objeto de estudio reportan información para el cálculo de los indicadores financieros, y el volumen de ingresos, permitiendo a través de este último determinar su tamaño.

Se considera en el estudio toda la población disponible en la base de datos de la Supercía que cumple con los criterios enlistados, por ello, no se establece una muestra.

En la Tabla 2 muestra las relaciones entre la estructura de capital y el nivel de información asimétrica. Al hacerlo, se construye la hipótesis de sus efectos sobre la estructura de capital desde el punto de vista de la teoría del Pecking Order. La hipótesis planteada hace referencia a que las empresas del sector alojamiento y servicios de comida, toman sus decisiones de endeudamiento de acuerdo con los lineamientos de la teoría de Pecking Order.

Tabla 2 Efectos de la asimetría de la información

\begin{tabular}{lc}
\hline Determinantes de la estructura de capital & Asimetría de la información \\
\hline Oportunidades de crecimiento & + \\
Tamaño & - \\
Edad & - \\
Tangibilidad del activo & - \\
\hline
\end{tabular}

Fuente: Cardone y Casasola, 2003 
El procesamiento de la información se estructuró en formato tabular para analizar la información de cada empresa, el tamaño de las mismas y sus indicadores financieros. El análisis estadístico respectivo se realiza utilizando el programa Microsoft Excel 2010.

La información utilizada proviene del estado de situación financiera y del estado de resultados de las empresas ecuatorianas del sector de alojamiento y servicios de comida, información comparable porque esta elaborado de acuerdo con las normas contables; la información también proviene de los indicadores financieros por año, publicados en el portal de información de la Superintendencia de Compañías.

Para organizar la información relevante de las variables, el estudio constará de un análisis descriptivo de las empresas en función de sus diferentes tamaños y actividad económica e involucra la revisión de indicadores financieros de endeudamiento y apalancamiento del sector; para cada uno de los ratios se ha calculado el valor promedio.

A partir de la Tabla 3, se puede observar la distribución de las empresas y como el porcentaje de microempresas ha aumentado de un período a otro, mientras que las empresas pequeñas no presentan mayor cambio, esto se debe a que el sector de alojamiento y servicios de comida es dinámico generando una mayor tasa de crecimiento de las empresas que se han adaptado a las necesidades del consumidor.
La proporción de las empresas medianas y grandes ha disminuido durante los años 2014 y 2015, cabe mencionar que esta situación se debe principalmente a factores externos como la caída del precio del petróleo, y a situaciones como el estancamiento económico del 2015, afectadando internamente a las empresas al disminuir el nivel de ventas, considerando que las grandes y medianas empresas de este sector se caracterizan por ofrecer servicios de lujo, viendose seriamente afectadas por los cambios de comportamiento del consumidor al preferir servicios al alcance de sus ingresos (Instituto Nacional de Estadísticas y Censos, INEC, 2017).

Las empresas pequeñas son representativas en este sector. Las grandes empresas apenas representan el $7 \%$ frente al $50 \%$ de las pequeñas empresas. El segundo lugar lo ocupan las medianas empresas con un $26 \%$ y le siguen las microempresas con $16 \%$.

Asi también, se observa que el $55 \%$ de las empresas son catálogadas como maduras al tener un tiempo de funcionamiento de 10 años en adelante; y 276 empresas que representan el $45 \%$ restante, pueden considerarse jóvenes o en proceso de consolidación con un promedio de 6 años de edad. Su actividad económica está divida en un $59 \%$ para las empresas que se dedican a la actividad de servicio de comida y el $41 \%$ restante se dedica a la actividad de alojamiento.

Tabla 3 Empresas por edad, tamaño y actividad económica

\begin{tabular}{|c|c|c|c|c|c|c|c|}
\hline Edad de la firma & \multicolumn{2}{|c|}{ No. Empresas } & \multicolumn{2}{|c|}{ Porcentaje } & \multicolumn{3}{|c|}{ Edad Promedio } \\
\hline Jóvenes o en proceso de consolidación & \multicolumn{2}{|c|}{276} & \multicolumn{2}{|c|}{45} & \multicolumn{3}{|c|}{6} \\
\hline Maduras & \multicolumn{2}{|c|}{338} & \multicolumn{2}{|c|}{55} & \multicolumn{3}{|c|}{19} \\
\hline \multirow{2}{*}{ Tamaño } & \multicolumn{2}{|c|}{2013} & \multicolumn{2}{|c|}{2014} & \multicolumn{2}{|c|}{2015} & \multirow[t]{2}{*}{ Promedio } \\
\hline & No. Empresas & $\%$ & No. Empresas & $\%$ & No. Empresas & $\%$ & \\
\hline Micro & 80 & 13 & 110 & 18 & 110 & 18 & $16 \%$ \\
\hline Mediana & 179 & 29 & 151 & 25 & 151 & 25 & $26 \%$ \\
\hline Grande & 61 & 10 & 35 & 6 & 35 & 6 & $7 \%$ \\
\hline Total & 614 & 100 & 614 & 100 & 614 & 100 & $100 \%$ \\
\hline Actividad Económica & & & & & & & Promedio \\
\hline Alojamiento & 254 & 41 & 254 & 41 & 256 & 42 & $41 \%$ \\
\hline
\end{tabular}

Nota. Tamaño de la empresa considerado de acuerdo con el Art. 106 del Reglamento del Código Orgánico de la Producción, Comercio e Inversiones [COPCI]. Edad de las empresas: Jóvenes o en proceso de consolidación $<10$ años; Maduras $>=10$ años

Fuente: Cardone y Casasola, (2003)

\section{Análisis de resultados}

El nivel de endeudamiento de las empresas del sector de alojamiento y servicios de comida durante el período 2013-2015 varió en promedio entre el $61 \%$ al $67 \%$, pero al analizar las empresas por tamaño se observa un mayor endeudamiento en las micro, pequeñas y medianas empresas con un índice por encima del 60\%; mientras que las grandes empresas en los tres años presenta un nivel de endeudamiento por debajo del $60 \%$. Las empresas pequeñas han superado a las medianas y grandes en su nivel de endeudamiento (Tabla 4)

Las pymes del sector alojamiento y servicios de comida del Ecuador tienen aproximadamente un $66 \%$ de concentración del endeudamiento en el corto plazo durante los años 2013-2015, lo cual puede ser riesgoso si existiera dificultades al generar recursos, ya que pueden ocasionar problemas financieros para cubrir sus obligaciones a corto plazo (Zeitun y Tian, 2007). 
Las empresas pequeñas y medianas tienen mayor concentración con índices superiores al $63 \%$, en las microempresas el índice oscila entre $48 \%$ y $52 \%$ en los años analizados, mientras que en las grandes empresas solo en el 2015 superó el $70 \%$.

Tabla 4 Indicadores de endeudamiento

\begin{tabular}{|c|c|c|c|c|c|}
\hline Indicadores & Empresas & 2013 & 2014 & 2015 & Promedio \\
\hline \multirow{5}{*}{ Nivel de endeudamiento ( \%) } & Microempresas & 62 & 74 & 63 & 66 \\
\hline & Pequeñas & 96 & 68 & 71 & 78 \\
\hline & Medianas & 62 & 62 & 61 & 62 \\
\hline & Grandes & 49 & 54 & 51 & 52 \\
\hline & Promedio & 67 & 65 & 61 & 64 \\
\hline \multirow{5}{*}{$\begin{array}{l}\text { Concentración del endeudamiento } \\
\text { en el corto plazo (\%) }\end{array}$} & Microempresas & 48 & 52 & 52 & 51 \\
\hline & Pequeñas & 67 & 67 & 65 & 67 \\
\hline & Medianas & 63 & 68 & 67 & 66 \\
\hline & Grandes & 59 & 67 & 72 & 66 \\
\hline & Promedio & 59 & 64 & 64 & 62 \\
\hline \multirow{5}{*}{$\begin{array}{l}\text { Concentración del endeudamiento } \\
\text { con entidades financieras ( } \%)\end{array}$} & Microempresas & 0 & 0 & 5 & 2 \\
\hline & Pequeñas & 5 & 0 & 5 & 3 \\
\hline & Medianas & 9 & 0,4 & 7 & 6 \\
\hline & Grandes & 11 & 1 & 5 & 6 \\
\hline & Promedio & 6 & 0 & 6 & 4 \\
\hline \multirow{5}{*}{$\begin{array}{l}\text { Concentración del endeudamiento } \\
\text { con proveedores ( } \%)\end{array}$} & Microempresas & 17 & 11 & 11 & 13 \\
\hline & Pequeñas & 24 & 20 & 21 & 22 \\
\hline & Medianas & 23 & 17 & 22 & 21 \\
\hline & Grandes & 19 & 20 & 27 & 22 \\
\hline & Promedio & 21 & 17 & 21 & 19 \\
\hline \multirow{5}{*}{ No. de veces que se gana el interés } & Microempresas & 0,01 & $-0,21$ & $-0,01$ & -0.07 \\
\hline & Pequeñas & 0,71 & 0,26 & 0,05 & 0,34 \\
\hline & Medianas & 1,59 & 0,83 & 0,86 & 1,09 \\
\hline & Grandes & 3,88 & 0,37 & 0,49 & 1,58 \\
\hline & Promedio & 1,55 & 0,31 & 0,35 & 0,74 \\
\hline
\end{tabular}

Fuente: Superintendencia de Compañias del Ecuador.

Elaboración: Autores.

Las obligaciones con instituciones financieras aumentaron en el 2015. La concentración de la deuda bancaria en el 2013 era de aproximadamente el $6 \%$ y en el 2014 disminuyó a menos del $1 \%$, para el 2015 este porcentaje aumentó nuevamente a $6 \%$. Este sector muestra que, a mayor tamaño de la empresa, mayor concentración de endeudamiento con instituciones financieras evidenciándose el problema que atraviesan las Mipymes para acceder a recursos a largo plazo y poder financiar sus proyectos, convirtiéndose en una desventaja frente a las grandes empresas. Esta situación denota principalmente la incomprensión que existe del funcionamiento de las micro, pequeñas y medianas empresas, respaldada por la ideología de que este tipo de empresas carecen de una adecuada gestión empresarial, situación que genera el problema de acceso a recursos (Zevallos, 2007).

El endeudamiento con los proveedores es la segunda opción de financiación de las empresas del sector de alojamiento y servicios de comida, este indicador oscila en promedio entre el $11 \%$ y el $27 \%$. En las microempresas el uso de los proveedores como fuente de generación de recursos decayó al pasar del 17\% en el 2013 al $11 \%$ en el 2015. La tendencia a disminuir el uso de financiación con proveedores fue similar en las pequeñas y medianas empresas, esto se debe a los incentivos que se están dando en la actualidad para acceder a préstamos bancarios, sin embargo, la financiación bancaria todavía presenta dificultades de acceso, producto de la asimetría de la información de este tipo de empresas y que no cuentan con mayores garantías que les permitan asumir los préstamos, siendo necesario financiarse a través de proveedores. En el caso de las medianas empresas esta disminución se debe al cierre de varias firmas en los periodos de estudio. Sin embargo, en las grandes empresas este porcentaje aumentó del $19 \%$ en el 2013 al $27 \%$ en el 2015 , como resultado de la necesidad de estar financiados con recursos que generan el menor costo a la empresa debido a la disminución en los niveles de ventas de sus servicios.

Durante los años 2013, 2014, 2015, las empresas del sector del alojamiento y servicio de comida no alcanzaron a cubrir los intereses con la utilidad operacional. En las 
microempresas esta situación fue demasiado difícil durante el período analizado con valores de -0.21 en el año 2014, ya que esto ocasiona un riesgo continuo, porque la empresa se ve obligada a mantener altos niveles de utilidades para ser productivos. Por otro lado, las empresas medianas y grandes arrojan datos de 1.59 y 3.88 respectivamente en el año 2013, lo que significa que las empresas contaban con 0,59 y 0,88 centavos para cumplir con los gastos de operación después de hacer frente a los pasivos financieros propios del giro del negocio.

Al relacionar la deuda con el patrimonio se aprecia que fue superior a 1 con tendencia a crecer (Tabla 5)

Los resultados en el apalancamiento financiero total para los tres años analizados son muy fluctuantes, esto se debe a la disminución del patrimonio ocasionada por las pérdidas reportadas de la operación de las empresas del sector de alojamiento y servicio de comida. Este indicador establece el grado de compromiso del patrimonio de la empresa con sus acreedores, la cual se presenta con niveles mayores en el corto plazo.

El apalancamiento financiero a corto plazo sigue una tendencia un poco más homogénea en las grandes empresas, por el contrario, en los tres años para las micro y pequeñas empresas tiene una tendencia al alza, mientras que en las medianas empresas se observó una disminución considerable entre los años 2013 al 2015.

Las empresas del sector de alojamiento y servicios de comida disminuyeron el apalancamiento con instituciones financieras desde el 2013 al 2015; las pequeñas y grandes empresas en el 2013 presentan mayor financiación que las micro y medianas empresas en el mismo año, esto quiere decir que menor será el riesgo de insolvencia.

Tabla 5 Indicadores de apalancamiento

\begin{tabular}{llcccc}
\hline Indicadores & Empresas & $\mathbf{2 0 1 3}$ & $\mathbf{2 0 1 4}$ & $\mathbf{2 0 1 5}$ & Promedio \\
\hline \multirow{4}{*}{ Apalancamiento financiero total } & Microempresas & 6,30 & 53,16 & 62,46 & 40,64 \\
& Pequeñas & 19,38 & 21,24 & 16,25 & 18,96 \\
& Medianas & 31,51 & 6,51 & 4,14 & 14,06 \\
& Grandes & 6,49 & 3,77 & 1,86 & 4,04 \\
& Promedio & 15,92 & 21,17 & 21,18 & 19,42 \\
\hline \multirow{4}{*}{$\begin{array}{l}\text { Apalancamiento financiero a corto } \\
\text { plazo }\end{array}$} & Microempresas & 5,67 & 9,00 & 10,92 & 8,53 \\
& Pequeñas & 5,43 & 10,79 & 9,57 & 8,60 \\
& Medianas & 11,22 & 4,35 & 2,94 & 6,17 \\
& Grandes & 1,21 & 2,82 & 1,41 & 1,81 \\
Apalancamiento financiero con & Promedio & 5,88 & 6,74 & 6,21 & 6,28 \\
\hline entidades financieras & Microempresas & 0,00 & 0,00 & 0,84 & 0,28 \\
& Pequeñas & 1,82 & 0,00 & 0,18 & 0,67 \\
& Medianas & 0,09 & 0,00 & 0,14 & 0,08 \\
& Grandes & 2,93 & 0,05 & 0,09 & 1,02 \\
\hline
\end{tabular}

Fuente: Superintendencia de Compañias del Ecuador.

Elaboración: Autores.

De acuerdo con el comportamiento de los diferentes tamaños de empresas en los períodos analizados se puede decir que tienen tendencia al Pecking Order a pesar de ciertas fluctuaciones en los resultados que se presentan a continuación. Según los datos las microempresas presentan:

- Un nivel de endeudamiento que varía entre el $49 \%$ y $96 \%$, pero poco a poco han ido disminuyendo estos valores a través de los tres años.

- La concentración de la deuda a corto plazo y con instituciones financieras incremento levemente.

- Una disminución leve de la concentración de la deuda con proveedores.

Las empresas pequeñas y medianas presentan una leve disminución, lo cual es bueno para confirmar la teoría de Pecking Order, que los empresarios prefieren en primer lugar apalancarme con recursos propios.

Se presenta disminución en:
- Nivel de endeudamiento

- Concentración de la deuda a corto plazo.

- Concentración de endeudamiento con instituciones financieras.

- Concentración de endeudamiento con proveedores.

Las empresas grandes presentan:

- Un incremento en el nivel de endeudamiento.

- Un aumento en la concentración de la deuda a corto plazo.

- Una disminución en el endeudamiento con instituciones financieras.

- Un crecimiento en la concentración de endeudamiento con proveedores.

El ROI (Rendimiento sobre la inversión) paso de su peor año en el 2013 con $-2,38 \%$ al mejor, con $0,25 \%$ en el 2015 aún cuando es muy leve. Las Mipymes tienen un 
comportamiento similar, mientras que las grandes empresas en el 2015 crecen con relación al 2013 (Tabla 6)

Las empresas del sector de alojamiento y servicios de comida tuvieron un rendimiento negativo en promedio para el período 2013, debido a que la rotación de activos es un poco alta y el margen de utilidad fue negativo. Al comparar el margen de utilidad neta y el ROI de las microempresas con las pequeñas, se puede notar que su indicador es menos volátil. El apalancamiento financiero esta por encima de dos en todos los años analizados, lo que originó que en los dos primeros años el resultado del ROI fuera negativo, pero en el 2015 presenta una tendencia positiva, con lo cual las empresas de este sector sufrieron las consecuencias de tener un favorable y desfavorable apalancamiento financiero.

Tabla 6 Índices del sistema de Du Pont

\begin{tabular}{|c|c|c|c|c|c|}
\hline Indicadores & Empresas & 2013 & 2014 & 2015 & Promedio \\
\hline \multirow{5}{*}{ Margen de utilidad neta ( \%) } & Microempresas & $-0,43$ & $-0,18$ & 0,06 & $-0,18$ \\
\hline & Pequeña & $-0,04$ & $-0,01$ & 0,04 & 0,00 \\
\hline & Medianas & $-0,03$ & 0,06 & 0,06 & 0,03 \\
\hline & Grandes & 0,02 & 0,1 & 0,1 & 0,07 \\
\hline & Promedio & $-0,12$ & $-0,01$ & 0,06 & $-0,02$ \\
\hline \multirow{5}{*}{ Rotación de activos totales (veces) } & Microempresas & 5,57 & 0,79 & 1,53 & 2,63 \\
\hline & Pequeña & 5,11 & 2,75 & 2,62 & 3,50 \\
\hline & Medianas & 2,13 & 2,29 & 2,19 & 2,20 \\
\hline & Grandes & 1,75 & 2,69 & 2,64 & 2,36 \\
\hline & Promedio & 3,64 & 2,13 & 2,25 & 2,67 \\
\hline \multirow{5}{*}{$\mathrm{ROI}=$ margen $\mathrm{x}$ rotación $(\%)$} & Microempresas & $-2,38$ & $-0,14$ & 0,09 & $-0,81$ \\
\hline & Pequeña & $-0,22$ & $-0,02$ & 0,11 & $-0,05$ \\
\hline & Medianas & $-0,06$ & 0,14 & 0,12 & 0,07 \\
\hline & Grandes & 0,04 & 0,27 & 0,25 & 0,18 \\
\hline & Promedio & $-0,66$ & 0,06 & 0,14 & $-0,15$ \\
\hline \multirow{5}{*}{$\begin{array}{l}\text { Apalancamiento financiero } \\
\text { (Activo/Patrimonio) }\end{array}$} & Microempresas & 7,30 & 54,16 & 63,46 & 41,64 \\
\hline & Pequeña & 20,38 & 22,31 & 17,25 & 19,98 \\
\hline & Medianas & 32,51 & 7,51 & 5,14 & 15,06 \\
\hline & Grandes & 7,49 & 4,77 & 2,86 & 5,04 \\
\hline & Promedio & 16,92 & 22,19 & 22,18 & 20,43 \\
\hline \multirow{5}{*}{$\begin{array}{l}\mathrm{ROE}=\text { margen } \mathrm{x} \text { rotación } \mathrm{x} \\
\text { apalancamiento }(\%)\end{array}$} & Microempresas & $-17,39$ & $-7,83$ & 5,67 & $-6,46$ \\
\hline & Pequeña & $-4,51$ & $-0,50$ & 1,81 & $-0,99$ \\
\hline & Medianas & $-1,84$ & 1,02 & 0,63 & $-0,12$ \\
\hline & Grandes & 0,29 & 1,26 & 0,72 & 0,77 \\
\hline & Promedio & $-5,86$ & $-1,51$ & 2,21 & $-1,70$ \\
\hline
\end{tabular}

Fuente: Superintendencia de Compañias del Ecuador.

Elaboración: Autores.

El ROI como indicador de la eficiencia con que las empresas de este sector utilizan sus inversiones sin tener en cuenta su estructura de capital, en promedio ha generado por sus activos una rentabilidad de $-0,15 \%$ que comparada con la concentración de endeudamiento con instituciones financieras que es de $4 \%$, se puede observar que presentan un apalancamiento financiero negativo, al ser el coeficiente del ROI inferior al endeudamiento financiero.

En cuanto al ROE mide la rentabilidad financiera, aunque el panorama es poco alentador, considerando que sus índices en promedio representan $-1,70 \%$, valor que no compensa la rentabilidad que espera obtener el inversionis- ta por el riesgo asumido al proporcionar sus recursos en esta actividad.

\section{Conclusión}

En este trabajo se ha estudiado la estructura de capital de las micro, pequeñas, medianas y grandes empresas del sector de alojamiento y servicios de comida con la finalidad de determinar la fuente de financiación utilizada, para ello se ha realizado un análisis financiero de las empresas mediante el uso de datos de panel y se han examinado las similitudes con las teorías Pecking Order y Trade Off, encontrando los siguientes hallazgos: 
- Las empresas del sector de alojamiento y servicios de comida poseen altos niveles de endeudamiento y se observa alta concentración en el corto plazo, sobresaliendo el endeudamiento con proveedores y en menor medida con instituciones financieras.

- Las microempresas tienen menor concentración de endeudamiento al corto plazo, y acuden menos a préstamos bancarios.

- Las pequeñas empresas se endeudan más pero con menor concentración en el largo plazo, además recurren frecuentemente al endeudamiento con proveedores.

- Las medianas empresas tienen un nivel de endeudamiento alto, siendo el segundo grupo de empresas con mayor concentración de endeudamiento con entidades bancarias.

- Las grandes empresas utilizan menos deuda, pero con mayor concentración con instituciones financieras y mayor palanca financiera a largo plazo.

- Las empresas muestran una rentabilidad negativa sobre el endeudamiento. Tradicionalmente la prueba empírica utilizada por la teoría Pecking Order se basa en una relación inversa entre la rentabilidad de la empresa y su apalancamiento, afirmando a través de los hallazgos financieros que la teoria del Pecking Order responde a la forma de financiación de este sector.

- Se comprueba la válidez empírica de las predicciones hechas por la teoría del Pecking Order con relación a otra variable que afecta la estructura de capital, tal como, el tamaño de la empresa, pues se observa en el análisis financiero que a mayor tamaño mayor acceso a recursos externos, principalmente por ser consideradas menos riesgozas y más atráctivas al existir poca o nula asimetría de la información, además de disponer de activos que le permiten hacer frente a sus obligaciones.

De acuerdo con los resultados obtenidos se puede afirmar que las empresas del sector de alojamiento y servicios de comida financian sus proyectos acorde al siguiente orden: 1. recursos patrimoniales, 2. pasivos a corto plazo, con alta concentración con proveedores y 3 . deuda financiera, en una proporción significativamente menor.

Este análisis refleja que los empresarios prefieren financiarse con recursos internos antes que perder su participación, lo que conllevaría a que se sacrifique el potencial crecimiento de estas empresas. No obstante, Deakins, Logan, y Steele, (2001) consideran que el optar por financiación interna no solamente es consecuencia de que el empresario no quiere perder el control de la empresa, sino a la falta de confianza de la banca en este tipo de negocios y además del poco interés de los inversionista, adicionamente para el caso de las Mipymes podría agregarse como limitante su capacidad de generar recursos y conseguir financiación que les permitan permanecer en el mercado y mejorar su desempeño.

Lo óptimo para estas empresas es contar con los recursos necesarios para hacer frente a los retos empresariales, más aún considerando que los consumidores de servicios son exigentes y requerirán un valor agregado para satisfacer su necesidad, por ello, lo importante no es preferir una determinada forma de financiación, sino lograr el acceso a recursos que beneficien a la empresa.

Para finalizar, se recalcan las limitaciones a las que están sometidas las conclusiones. En primer lugar, se trata de información obtenida a través de los indicadores financieros de las empresas del sector en un momento puntual, cuyos datos fueron recopilados de la Superintendencia de Compañías. En segundo lugar, el tipo de análisis realizado presenta limitaciones en cuanto a causalidad, pues no permite comprobar con certeza las circunstancias que llevaron a los empresarios a tomar determinadas decisiones que derivaron los resultados expuestos. Por ello, es necesario posteriores trabajos que analicen las elecciones de financiación por cada empresa para lograr resultados más certeros, además de estudiar el efecto de la asimetría de la información en el acceso a recursos externos.

Así también, es importante se efectúen nuevos estudios que amplíen el análisis de las particularidades de cada sector empresarial, así como el apoyo que brinda el estado en el financiamiento de las empresas a través de las políticas públicas.

\section{Referencias Bibliográficas}

Ana Milena Medina, O., José Daniel Salinas, R., Lina María Ochoa, B., y Carlos Andrés Molina, G. (2012). La estructura financiera de las empresas manufactureras colombianas, una mirada econométrica, macroeconómica y financiera. Perfil de Coyuntura Económica, O(20), 155-176. Descargado de http://aprendeenlinea - udea.edu.co/revistas/index.php/ coyuntura/article/view/18189

Barona Zuluaga, B., y Rivera Godoy, A. (2010). Aspectos conceptuales y empíricos de la financiación de nuevas empresas en colombia. Cuadernos de Administración, 81-97. Descargado de http: / /www.redalyc.org/articulo . oa?id=225017552006

Barona Zuluaga, B., y Rivera Godoy, J. A. (2012). Análisis empírico de la financiación de nuevas empresas en colombia. Revista Innovar, 22(43), 5-18.

Camino Mogro, S., y Guale Córdova, A. (2017). Estudios sectoriales: Alojamiento y servicios de comida. dirección nacional de investigación y estudios de la superintendencia de compañías, valores y seguros del ecuador. Descargado de ://www. supercias -gob.ec/portalscvs/

Cardone Riportella, C., y Casasola, M. J. (2003, Jul). What do we know about the financial behaviour of the Spanish SME?: an empirical analysis. Descargado de https://e-archivo.uc3m.es/handle/ $10016 / 79$ ? show=full

Instituto Nacional de Estadísticas y Censos, INEC. (2017). Panorama laboral y empresarial del ecuador. Des- 
cargado de http://www.ecuadorencifras . gob.ec

Isaac, F., Flores, O., y Jaramillo, J. (2010). Estructura de financiamiento de las pymes exportadoras mexicanas. análisis de politica de deuda. Observatorio de la Economía Latinoamericana(135). Descargado de http://www.eumed.net/cursecon/ ecolat/mx/2010/ifj.htm

Jensen, M. C. (1986). Agency costs of free cash flow, corporate finance and takeovers. American Economic Review, 76(2), 323-329.

Loncan, T., y Caldeira, J. (2014, abr.). Estrutura de capital, liquidez de caixa e valor da empresa: estudo de empresas brasileiras cotadas em bolsa. Revista Contabilidade e Finanças, 25(64), 46-59. Descargado de http: / / www.revistas.usp.br/rcf/ article/view/80749 doi: 10.1590/S1519 $-70772014000100005$

Mejía Amaya, A. (2015). The capital structure in the company: its contemporary study. Revista Finanzas y Política Económica, 5(2), 141-160. Descargado de https://editorial.ucatolica.edu.co/ ojsucatolica/revistas_ucatolica/ index.php/RFYPE/article/view/446

Modigliani, F., y Miller, M. (1963). Corporate income taxes and the cost of capital: A correction. The American Economic Review. Descargado de https://www2.bc.edu/ thomas-chemmanur/phdfincorp/ MF 891\%20papers/MM1963.pdf

Moreira Da Silva, C. A., y Rodríguez Sanz, J. A. (2006). Contraste de la Teoría del Pecking Order versus la Teoría del Trade-Off para una Muestra de Empresas Portuguesas. Universidad de Salamanca (España). Facultad de Economía y Empresa. Descargado de https://gredos.usal.es/jspui/ handle/10366/75182

Pettit, R. R., y Singer, R. F. (1985). Small business finance: A research agenda. Financial Management, 14(3), 47-60. Descargado de http: //www .jstor.org/stable/3665059

Rajan, R. G., y Zingales, L. (1995). What do we know about capital structure? some evidence from international data. The Journal of Finance, 50(5), 1421-1460. Descargado de https:// onlinelibrary.wiley.com/doi/abs/ $10.1111 / j .1540-6261.1995 . t b 05184 . x$ doi: 10.1111/j.1540-6261.1995.tb05184.x

Rivera Godoy, J. A. (2007, Dec). Estructura financiera $y$ factores determinantes de la estructura de capital de las pymes del sector de confecciones del Valle del Cauca en el período 2000-2004. 1., 20(34). Descargado de http://revistas.javeriana.edu.co/ index.php/cuadernos_admon/article/ view/4050

Saona Hoffmann, P. (2009). Estructura de capital de las empresas que cotizan en la bolsa de valores chilena un análisis comparativo basado en la teoría.

Shyam-Sunder, L., y C. Myers, S. (1999, feb). Testing static tradeoff against pecking order models of capital structure1This paper has benefited from comments by seminar participants at Boston College, Boston Unsiversity, Dartmouth College, Massachusetts Institute of Technology, University of Massach. Journal of Financial Economics, 51(2), 219-244. Descargado de http: / / linkinghub.elsevier.com/ retrieve/pii/S0304405X98000518 doi: 10.1016/S0304-405X(98)00051-8

Watson, R., y Wilson, N. (2002). Small and medium size enterprise financing: A note on some of the empirical implications of a pecking order. Journal of Business Finance \& Accounting, 29(3-4), 557-578. Descargado de https://onlinelibrary.wiley.com/ doi/abs/10.1111/1468-5957.00443 doi: 10.1111/1468-5957.00443

Zambrano Vargas, S. M., y Acuña Corredor, G. A. (2011). Estructura de capital. Evolución teórica. Criterio Libre(15), 81-102. Descargado de https://dialnet.unirioja.es/ servlet/articulo? codigo=3815888

Zata-Potziouris, P. (2011). The financial structure and performance of owner-managed family firms: Evidence from the uk economy.

Zeitun, R., y Tian, G. G. (2007). Capital structure and corporate performance: evidence from Jordan. Research Online, 1(4), 3. Descargado de https : / / ro.uow. edu.au/aabfj/vol1/iss $4 / 3$

Zevallos, E. (2007). Restricciones del entorno a la competitividad empresarial en américa latina. costa rica. Fundes.

Aceptado: 7 de septiembre de 2018 
